\section{Es ist genug für alle da}

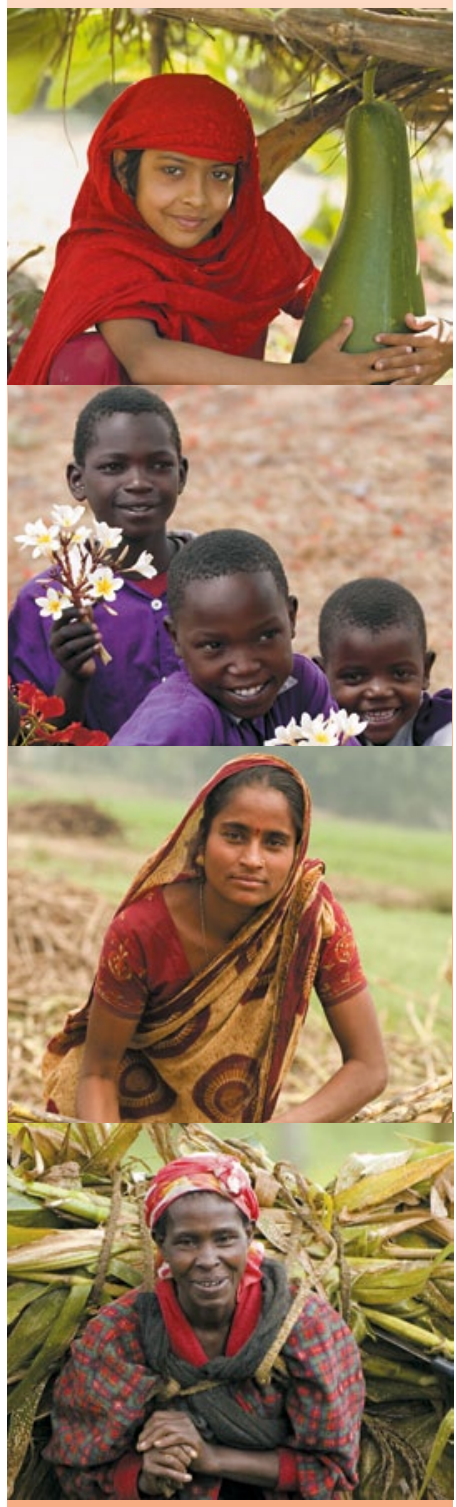

Fotos: Christof Krackhardt

... wenn wir miteinander teilen.

\section{Teilen Sie mit.}

\section{Postbank Köln}

500500500

BLZ 37010050

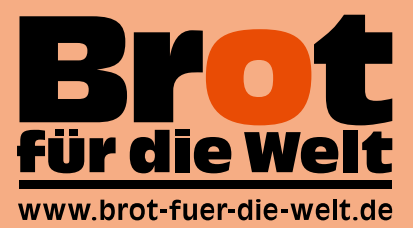

Berufsverband der Palliativmediziner

in Westfalen-Lippe e.V.

\title{
Palliativmedizin für Fortgeschrittene
}

— Gemäß der Weiterbildungsordnung der Landesärztekammern und des Curriculums zur Zusatzweiterbildung Palliativmedizin der Bundesärztekammer (BÄK) und der Deutschen Gesellschaft für Palliativmedizin (DGP) bietet die Gesellschaft für Algesiologische Fortbildung in Kooperation mit dem Palliativzentrum am evangelischen Krankenhaus Herne, der Klinik für Schmerz- und Palliativmedizin am St. Marien-Hospital in Lünen, der Akademie für Palliativmedizin, Palliativpflege und Hospizarbeit (APPH) im Ruhrgebiet sowie den Universitätskliniken Greifswald und Rostock Fallseminare und Aufbaukurse zur Erlangung der Zusatzbezeichnung Palliativmedizin an.

Ziele der Kursweiterbildung sind die Sensibilisierung für die besondere Haltung, die palliativmedizinisches Handeln trägt, und ein geschärftes Bewusstsein für die über rein medizinische Fragestellungen hinausgehenden psychosozialen und spirituellen Aspekte der existenziell bedrohten Patienten und ihrer Angehörigen. Palliativmedizin vollzieht sich im multiprofessionellen Team (aus Ärzten, Pflegenden, Psychosozialen Berufen und Seelsorge) unter Integration der ehrenamtlichen Mitarbeiter.
Die Fallseminare bzw. Aufbaukurse zur Erlangung der Zusatzbezeichnung „Palliativmedizin“ gliedern sich in drei Module à 40 Stunden.

\section{Modul 1 und 2}

16.-20. Juni 2012 und 21.-25. Juni 2012

Ostseehotel Dierhagen

Wiesenweg 1, 18347 Dierhagen,

Telefon: 038226 - 51-0, Fax: 038226 - 51-871

\section{Modul 3}

12.-16. Dezember 2012

Haus der Kirche

Grüner Winkel 10, 18273 Güstrow,

Telefon: 03843 - 21 78-0, Fax: 03843 - 21 78-58

Geleitet werden die Kurse von Dr. med. W. Diemer, Palliativzentrum EvK Herne, ex Universitätsklinikum Greifswald (E-Mail: diemer@ uni-muenster.de) und Dr. med. E. A. Lux, Klinik für Schmerz- und Palliativmedizin, St.-Marien-Hospital Lünen (E-Mail: sp@klinikumluenen.de). Zertifiziert werden sie von der Landesärztekammer Mecklenburg-Vorpommern mit jeweils 40 Kreditstunden - und sind für die Weiterbildung zur Erlangung der Zusatzbezeichnung Palliativmedizin anerkannt.

\section{Fort- und Weiterbildung großgeschrieben}

\section{"Angewandte Schmerztherapie und Palliativ- medizin" ist Organ unseres Verbandes}

\begin{abstract}
- Mit der Website www.bv-palliativmediziner.de bietet der Berufsverband der Palliativmediziner Westfalen-Lippe e. V. seinen Mitgliedern und interessierten Kollegen viel an Informationen rund um die palliativmedizinische Versorgung in Westfalen-Lippe. Als Partner für Kommunikation konnten wir darüber hinaus die Zeitschrift „Angewandte Schmerztherapie und Palliativmedizin" gewinnen, die nun als Organ unseres Berufsverbandes in jeder Ausgabe auf diesen Seiten zeitnah über aktuelle Entwicklungen der Palliativmedizin und spezielle Angebote der Fort- und Weiterbildung informiert. Dadurch erreichen wir
\end{abstract}

nicht nur unsere Mitglieder, sondern fördern bundesweit den Austausch zwischen uns Palliativmedizinern ebenso wie mit interessierten Ärzten, Pflegenden, Physiotherapeuten und Psychologen.

Berichten Sie über Ihre Erfahrungen in der praktischen Patientenversorgung, sprechen Sie uns auf Themen an, die aus Ihrer Sicht wichtig sind, seien Sie konstruktiv kritisch. Wir freuen uns über Ihre Unterstützung!

Dr. Eberhard A. Lux für den Berufsverband und Doris Berger für die Redaktion der Zeitschrift „Angewandte Schmerztherapie und Palliativmedizin" 